\title{
RISK EVALUATION IN A TRANSPORTATION SYSTEM
}

\author{
F. RUSSO \& A. VITETTA \\ Department of Computer Science, Mathematics, Electronics and Transportation, Mediterranean University of Reggio \\ Calabria, Italy, Feo di Vito, Reggio Calabria, Italy.
}

\begin{abstract}
Methods for planning and managing a transportation system in an urban area, when exogenous events occur and/or in emergency conditions, have received little attention from transportation system researchers, research institutions and journals. Models and algorithms specified and calibrated for ordinary conditions cannot be directly applied in emergency conditions. In this paper, the main problems developed concern: formalization of the risk problem in a transportation system with an improvement over consolidated quantitative risk analysis models; specification of a system of models for evacuation design and simulation, and in the particular case of path design in emergency conditions; the application of the model to simulate a real system for exposure reduction. The application was developed for an Italian town of about 9,000 inhabitants which was chosen as it has a similar number of potential evacuees to the majority of cases in real events when entire small towns, parts of a city or big buildings had to be evacuated. The curve between vulnerability and exposure is quantitatively represented.

Keywords: evacuation, network design, risk analysis, simulation.
\end{abstract}

\section{INTRODUCTION}

This paper proposes methods for the simulation and design of a transportation system under conditions of safety and/or security. Safety and security problems are connected with events that generate emergency conditions, such as the 9/11 attack, the Atocha station bombings and the Asian tsunami.

Methods for planning a transportation system in an urban area when exogenous events affecting the system occur and/or in emergency conditions, have received little attention from transportation system researchers, institutions and journals. Models and algorithms specified and calibrated for ordinary conditions cannot be directly applied in emergency conditions. When an exogenous event occurs, non-informed users move on the network with behaviour that is different with respect to ordinary situations: they know neither system congestion nor reliability in real time, as the system is in an exceptional condition; and even if the user has knowledge about his/her optimum, the user optimum, in general, differs from the system optimum and from the community (general public) optimum. If the system is monitored and designed in real time, a subset of users, who knows the system congestion and reliability in real time, is informed. The user optimum could be similar to the system optimum and the general public optimum.

In these conditions, public decision makers must predict with transportation models (implemented by the system analyst) the effects of implementing their measures upon the system and how these measures may interact (implemented by operational forces) with the individual reactions of the users served by the transport system in question. System analysts implement a decision support system in order to evaluate in a short time the effect of the strategy that has to be implemented in the system; operational forces (military, fire brigade, etc.) organize the control and emergency services, and operate in direct contact with the users.

Emergency conditions related to safety and/or security problems in a transportation system can be activated by exogenous events (power failure, radiation leak, etc.) or by endogenous events to the transportation system (dangerous emissions, etc.).

The events that generate emergencies have an intensity level $x$ (the intensity level can be defined with a probabilistic curve $p$, where for each intensity level a probability that it happens is defined). 
The development of models for emergency conditions in transportation system has not received much attention in the literature. Only specific aspects are treated concerning large-scale emergencies when a nuclear event occurs [1], in urban systems when general hazards occur [2,3] and in buildings during fires $[4,5]$. In general, there is no systematic analysis of the general risk theory applied in the transportation system and very often the vulnerability and exposure in the transportation system are considered as similar variables, or in other cases the exposure variables are treated as vulnerability variables. When an event occurs in a system or is bound to happen in the short term, evacuation measures must be applied and in some cases have to be designed in real time. Models and algorithms specified and calibrated in ordinary conditions [6-9] cannot be directly applied in emergency conditions.

The emergency plan, in general, is defined considering two elements: each person knows exactly what to do (information); each person follows instructions exactly (coordination and organization). The current assumptions are that the emergency plan is well coordinated and organized and the information is optimal. But who guarantees that: the available choice set for the authorities and operational forces is good, where the choice set is defined in terms of information, coordination and organization? Is the choice made the best? And then are the assumptions true?

In real conditions, very often, to verify the quality of an evacuation strategy, a real simulation on the real system is tested. This approach is very close to real conditions but is costly in terms of money, organization and people involved. During real experimentation if some of the scenario configurations have to be modified, a new real evacuation has to be organized. In real conditions, also some new users could be present in the system and a priori experimentation is impossible.

Transportation risk analysis consists in developing a quantitative estimate of risk based on engineering assessments and possible mathematical techniques for combining estimates of incidents, consequences and frequencies [10]. In this paper, transportation risk analysis is proposed through a simulation approach with quantitative models where real conditions are reproducible on a computer. Computer simulation is cheaper than real simulation in terms of cost and time, and different strategies and scenarios can be tested. Real simulation may be useful to calibrate some parameters. For new users, some hypotheses can be made; stochastic models may help in this direction.

This paper is developed with the following main objectives:

(a) to formalize the risk problem with clear diversification (for the consequences) in the definition of the vulnerability and exposure in a transportation system; thus the paper gives improvements with respect to consolidated quantitative risk analysis models, especially transportation risk analysis models;

(b) to specify a system of models for evacuation design and simulation;

(c) to specify a model for path design in emergency conditions;

(d) to apply the model to simulate a real system for exposure reduction.

In relation to the proposed objectives in this paper:

(a) a general framework is reported in Section 2, with specific methods and models, to analyze urban transportation system performances in emergency conditions when exogenous phenomena occur, and for the specification of the risk function;

(b) a formulation of the general evacuation problem in the standard simulation context of a 'what to' approach and 'what if' approach is reported in Section 3;

(c) a specific formulation of the path design problem inside the general evacuation problem is reported in Section 4; 
(d) in Section 5, experimentation of a 'what if' approach is reported; the models are applied to simulate a real transportation system.

Some conclusions and indications for research developments are reported in Section 6.

\section{RISK FUNCTION}

Risk can be defined as a cardinal measure of potential economic loss, human injury or environmental damage in terms of both incident probability and magnitude of the loss, injury, or damage [10]. Risk has to be assessed in all its components (probability, vulnerability and exposure) and a numerical value has to be obtained. This value can be considered a cardinal measure of the safety and security level. An increase in the risk level is equivalent to a reduction in safety and security. A general formulation for risk assessment in all its components has to be developed.

Following the chemical research approach, two types of risks can be defined: individual risk and societal risk. Individual risk is associated with a particular person or at a particular location; societal risk is associated with an activity of a particular population.

In the literature [10], risk assessment is proposed by means of quantitative analysis defining:

- for individual risk,

(A) risk level that gives the risk level for a person at a particular location;

(B) the average individual risk that gives the average risk to all the population;

(C) the maximum individual risk that illustrates the highest risk to any one individual of a population;

- for societal risk,

(D) the societal risk level that gives the total risk level associated with an activity of a particular population.

Moreover in [10], risk assessment is proposed by means of geographic (map) analysis:

- for individual risk,

(E) the individual risk contour that illustrates the geographical distribution of individual risk;

- for societal risk,

(F) the societal risk curve that illustrates the curve of the probability and the consequences of the total risk associated with an activity of a particular population.

Risk above a certain level (in one or more indicators) is considered intolerable and unjustified, and some measures have to be introduced to prevent the risk exceeding a predefined risk level, named intolerable risk. The risk limit is not defined by scientific calculation but by observation of what society at present tolerates [11]. The change in intolerable risk over the years, combined with recent events, shows how the risk limit for society changes in time.

Individual intolerable risk is defined as a maximum level that the individual accepts for the individual risk level indexes. Over these values there is an alarm level and below these values an acceptable level. The definition of individual intolerable risk can be extended to societal intolerable risk, but there are some conceptual difficulties in defining this limit. This is due to the complexity of societal intolerable risk, as there is difficulty allowing different types of events at the same level. 
In the following subsections, the consolidated societal risk definition will be recalled and a general formulation of indicators for individual and societal risk will be proposed.

\subsection{Definitions and reference formulation to evaluate societal risk}

Starting from the definition of risk by which it depends on the probability (or the frequency) that the event occurs and on the magnitude of the loss, injury or damage, in a simplified version societal risk $R$ can be defined as:

$$
R=P M
$$

where $P$ is the probability that an emergency event occurs; $M$ is the magnitude defined as a cardinal measure of the consequences for a particular population.

Equation (1) can be also written in the form:

$$
R=P V N
$$

where $M=V N ; V$ represents the vulnerability and $N$ the exposure.

In the literature, there is no clear distinction between vulnerability and exposure, especially in transportation systems. Hence in this paper, we state the definition straightaway and propose a mathematical formulation.

The vulnerability of the system can be defined as the resistance of the infrastructure (material and immaterial) when the emergency occurs. Thus, it is related only to the resistance of the infrastructure during an emergency and there is no dependence on demand. Vulnerability can be considered as an indicator in the interval $[0,1]$ that shows the vulnerability of the system when the event happens: zero means that the resistance is infinite; one means that the resistance is null. Vulnerability is a supply characteristic. Vulnerability can be direct or inducted: the resistance connected to the first event is direct; the resistance connected to the events generated from the first is induced. Examples of vulnerability are: the degree of resistance of a bridge when an emergency occurs; the period of time during which a data network and servers function properly before a crash. Vulnerability could also be connected to buildings that collapse on road surfaces or to traffic lights at junctions that are out of order due to an event.

The exposure of the system can be defined as the equivalent homogeneous weighted value of people, goods and infrastructure affected during and after an event. Exposure is a demand and demand/supply interaction characteristic. An example of exposure is the number of users in the system area who could die if not evacuated when an emergency occurs. To use the same example as above, exposure is also the value of the bridge and/or the buildings that collapse on the road surface and also the number of people on the bridge or inside the buildings and again the number that can be evacuated.

In accordance with these definitions, the congested (real) travel time between two points in a system is a proxy indicator of exposure as it is a result of a demand/supply interaction. The connectivity of two points in terms of topology or the free flow travel time is a proxy indicator of the vulnerability characteristic since it does not depend on demand. In an emergency, the congested travel time is completely different from the free flow travel time: it is the congested time, which has to be considered.

Considering eqn (1), two types of measures for risk reduction may be defined (Fig. 1):

- prevention, which consists in reducing the level of $P$;

- protection, which consists in reducing the level of $M$.

Currently, the reduction of $\mathrm{P}$ is possible only for some kinds of events, which occur in relation to human activities (power failure, radiation leak, hazardous freight, etc.) and is the main objective 


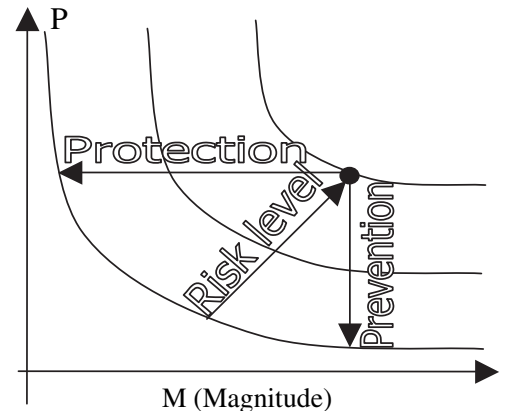

Curves with constant risk level $R$

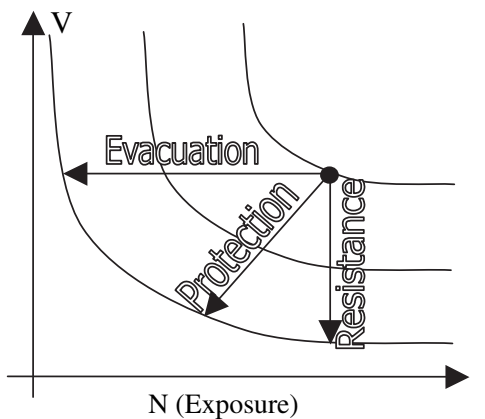

Curves with constant magnitude level $M$

Figure 1: Possible measures for societal risk reduction.

of safety planners. Other kinds of events (bomb attack, etc.) are the main objectives of security planners (military, etc.).

The magnitude $(M=V N)$ can be reduced with two classes of measures (Fig. 1):

- resistance, which consists in reducing the level of $V$;

- evacuation, which consists in reducing the level of $N$.

In line with previous definitions, resistance means the increase in the ability of the infrastructure (transport and otherwise) to withstand events. Evacuation consists in reducing the number of users and goods that can experience negative effects when emergency events occur.

\subsection{Proposed generalized formulation for risk}

In this paper, a generalized formulation is proposed for societal risk assessment that goes beyond the traditional formulation where vulnerability and exposure are considered constant throughout the area studied. Having defined an emergency event $E$ (e.g. a radiation leakage in a nuclear power station) in an area $T$ (land around the power station), event $E$ may occur in $T$ with an intensity level in the range $L_{E}$ (e.g. the intensity of radiation emissions between two prefixed values), in the time slice $\Delta$ (e.g. in the next 2 years).

For each event, a probability function $p(x, y, z)$ can be defined where $x, y$ and $z$ are respectively the variables for the intensity level in the range $L_{E}$, the area $T$ and the time slice $\Delta$. The function $p$ is a probability function and can be defined as:

$$
\int_{x \in L_{E}} \int_{y \in T} \int_{z \in \Delta} p(x, y, z) \mathrm{d} z \mathrm{~d} y \mathrm{~d} x /\left(\int_{x \in L_{E}} \int_{y \in T} \int_{z \in \Delta} \mathrm{d} x \mathrm{~d} y \mathrm{~d} z\right)=P
$$

with the constraint $P \in[0,1]$.

$P$ is the probability level that at least an event $E$ may occur in an area $T$, with an intensity level in the range $L_{E}$, in the time slice $\Delta$ and can be interpreted as the average value of the probability, considered constant with respect to $x, y$ and $z$. This hypothesis is not realistic. Hence the traditional model cannot be used and this new formulation is proposed.

The function $p$ is a probability function and the value $p(x, y, z) \mathrm{d} z \mathrm{~d} y \mathrm{~d} x$ is the probability that the event $E$ happens with an intensity level between $x$ and $x+\mathrm{d} x$ in the surface area $\mathrm{d} y$, around point $y$ in the time slice between $z$ and $z+\mathrm{d} z$. 
For natural events, the historical data relative to the events allow a frequency function to be calculated for each point of the land (or each homogeneous land unit), for each time period related to the return period of the event, and for a set class of intensity level. The frequency function can be used as the experimental data to compare with a theoretical probability function. A statistical test has to be used to verify whether the theoretical function is statistically equal to the experimental data.

For industrial events, a predictive model can be considered in order to generate an estimated probabilistic function. In this case a predictive model has to be used for two reasons: the historical data are very poor; industrial events can be predicted with quantitative models.

The level of magnitude that produces an event $E$ with intensity level $x$ at point $y$ of $\mathrm{T}$ is $M(x, y)$, given by the product of:

$$
M(x, y)=V(x, y) N(x, y)
$$

where

- vulnerability $V(x, y)$ is the capacity of infrastructure (buildings, roads, bridges, etc.) at point y of $\mathrm{T}$ not to withstand event $\mathrm{E}$ with intensity level $\mathrm{x}$; in terms of measurement units, the vulnerability is in the range $(0,1)$ and

- it is 0 if the infrastructure at point $y$ of $T$ suffers no damage from event $E$ with intensity level $x$;

- it is 1 if the infrastructure at point $y$ of $T$ is completely destroyed by event $E$ with intensity level $x$;

- exposure $N(x, y)$ is the equivalent homogeneous weighted value of people, goods and infrastructure at point $y$ of $T$ affected during and after the event occurring with intensity level $x$ in the range $L_{E}$; in terms of measurement units, the exposure is an equivalent value of damage for people, goods and infrastructure.

With the given definitions and notations (eqns (3) and (4)), assessments can be made with a set of indexes:

- for individual risk,

(A) the individual risk level, $R_{L_{E}, T, \Delta}^{i}(y)$, for event $E$, at point $y$ of area $T$, with intensity level in the range $L_{E}$, in a time slice $\Delta$, can be defined as:

$$
R_{L_{E}, T, \Delta}^{i}(y)=\int_{x \in L_{E}} \int_{z \in \Delta} M(x, y) p(x, y, z) \mathrm{d} z \mathrm{~d} x /\left(\int_{x \in L_{E}} \int_{z \in \Delta} \mathrm{d} x \mathrm{~d} z\right)
$$

(B) the average individual risk level, $R_{L_{E}, T, \Delta}^{a}(y)$, for event $E$, in area $T$, with intensity level in the range $L_{E}$, in a time slice $\Delta$, can be defined as:

$$
R_{L_{E}, T, \Delta}^{a}=\int_{y \in T} R_{L_{E}, T, \Delta}^{i}(y) \mathrm{d} y /\left(\int_{y \in T} \mathrm{~d} y\right)
$$

(C) the maximum individual risk level $R_{L_{E}, T, \Delta}^{m}(y)$, for event $E$, in area $T$, with intensity level in the range $L_{E}$, in a time slice $\Delta$, can be defined as:

$$
R_{L_{E}, T, \Delta}^{m}=\max _{y \in T} R_{L_{E}, T, \Delta}^{i}(y)
$$


- for societal risk,

(D) the general cardinal measure of societal risk for event $E$ in area $T$, with intensity level in the range $L_{E}$, in a time slice $\Delta$, can be defined as:

$$
\begin{aligned}
R_{L_{E}, T, \Delta} & =\int_{y \in T} R_{L_{E}, T, \Delta}^{i}(y) \mathrm{d} y /\left(\int_{x \in L_{E}} \int_{y \in T} \int_{z \in \Delta} \mathrm{d} x \mathrm{~d} y \mathrm{~d} z\right) \\
& =\int_{x \in L_{E}} \int_{y \in T} \int_{z \in \Delta} M(x, y) p(x, y, z) \mathrm{d} z \mathrm{~d} y \mathrm{~d} x /\left(\int_{x \in L_{E}} \mathrm{~d} x\right)\left(\int_{y \in T} \mathrm{~d} y\right)\left(\int_{z \in \Delta} \mathrm{d} z\right)
\end{aligned}
$$

The general measure for the societal risk is equal to the average individual risk level. Considering eqn (4), societal risk can be expressed as:

$$
R_{L_{E}, T, \Delta}=\int_{x \in L_{E}} \int_{y \in T} \int_{z \in \Delta} V(x, y) N(x, y) p(x, y, z) \mathrm{d} z \mathrm{~d} y \mathrm{~d} x /\left(\int_{x \in L_{E}} \mathrm{~d} x \int_{y \in T} \mathrm{~d} y \int_{z \in \Delta} \mathrm{d} z\right)
$$

Equation (2), considered for risk assessment, is a particular case of eqn (5) under several restrictive hypotheses.

In the hypothesis of

- vulnerability $V(x, y)$, constant with respect to $T$ and in the range $L_{E}$, and equal to $V$;

- exposure $N(x, y)$, constant in $T$ and in the range $L_{E}$, and equal to $N$;

societal risk assumes the simple form:

$$
R_{L_{E}, T, \Delta}=V N \int_{x \in L_{E}} \int_{y \in T} \int_{z \in \Delta} p(x, y, z) \mathrm{d} z \mathrm{~d} y \mathrm{~d} x /\left(\int_{x \in L_{E}} \mathrm{~d} x \int_{y \in T} \mathrm{~d} y \int_{z \in \Delta} \mathrm{d} z\right)
$$

Considering eqn (3), the risk reported in eqn (6) is equal to:

$$
R_{L_{E}, T, \Delta}=P V N
$$

Equation (7) is equal to eqn (2), but they are equivalent only in the particular hypothesis described.

\section{MODELS FOR EVACUATION}

In this section, a general procedure for evacuation simulation and design is reported (Section 3.1), considering briefly the areas of interest of different research sectors. In Sections 3.1 and 3.2, the areas of interest of transportation research concerning simulation and design models of the transport system are reported in detail.

\subsection{Procedure for the evacuation plan}

The drawing up of an evacuation plan for an urban area requires a study of the problems concerning land use and the disaster. The complete procedure for developing an evacuation plan can be subdivided into the following variously connected phases:

1. the study of the infrastructure and land use and definition of objectives and constraints;

2. the generation of 
2.1 sub-scenarios for supply;

2.2 sub-scenarios for demand;

3. probability and vulnerability evaluation;

4. model for

4.1 supply;

4.2 demand;

4.3 supply-demand interaction;

5. evaluation of

5.1 exposure;

5.2 risk acceptance.

In Fig. 2, a system of models and relative functional connections are represented, which may be proposed for testing evacuation plans by means of quantitative methods.

1. The first phase consists in studying the area as follows: the area is delimited and defined as that area comprising the transport system in question where most of the effects of the planned interventions are believed to take place; homogeneous areas are identified; zoning and a minimal road network

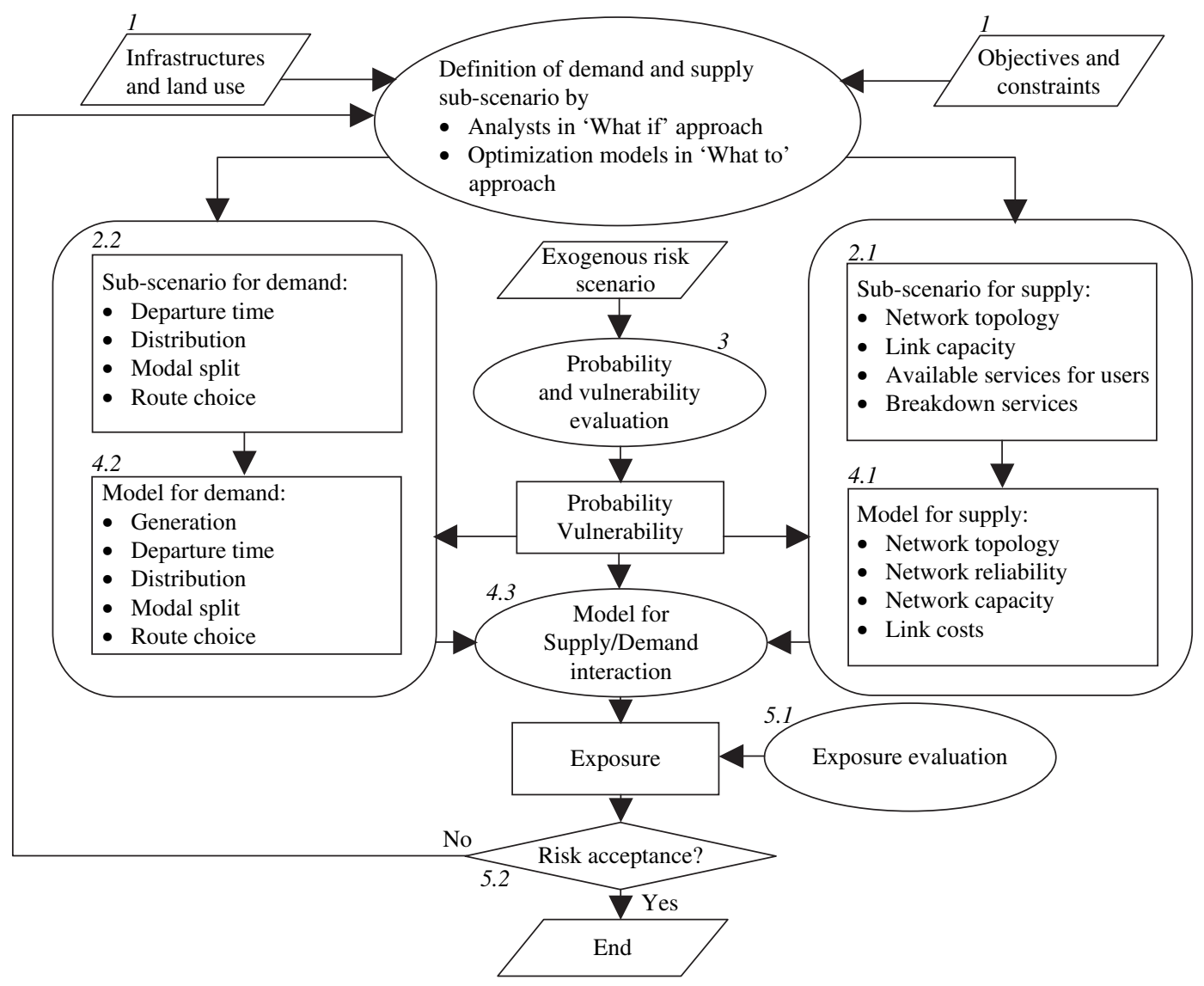

Figure 2: The procedure for the evacuation plan and relevant links. 
plan are developed. The objectives for users and the community, the general technical and financial constraints are defined.

2. The second phase consists in generating the demand-and-supply sub-scenario in relation to land use, the infrastructural network and available services in relation to the objectives and constraints of the general problem. Two different approaches can be considered:

- the 'what if' (or simulation) approach where the analysts, in relation to their experience, generate the scenario and verify its level of optimality (reported in Section 3.1);

- the 'what to' (or optimization) approach where the analyst builds models that self-generate the optimal scenario (reported in Section 3.2).

With evacuation simulation, a defined configuration for the transportation system is simulated. In a simulation approach, the configurations of the different scenarios are defined with the experience of the analyst, and the quality of the configurations is evaluated with some indicators.

3. The 'what to' approach is more reliable and contains the solution generated with the 'what if' approach; if an optimal solution of the problem has to be obtained, the 'what to' can be applied only in a subset of transportation problems. One of these problems is proposed in Section 4 to obtain the best path in evacuation. Only the 'what if' approach can be used in a large set of transportation problems. The third phase involves definition of the exogenous risk scenario and evaluation of probability and vulnerability in relation to the considered event that generates a risk. These aspects are studied in other fields than transportation and in these contexts are considered as external inputs.

4. The fourth phase involves supply, demand and supply-demand interaction analysis.

In relation to the sub-scenario for supplying the network topology, link capacity, available services for users and breakdown services are defined. The supply model analysis is carried out as follows:

- definition of the network topology in terms of connection between the different links and services;

- verification of the exogenous and endogenous disturbances on the transport network in order to evaluate network reliability;

- definition of link capacity relative to the different category of users;

- definition of link cost (for the static or pseudo-dynamic approach) or (for the within-day dynamic approach) functions relative to the different category of users.

In relation to the sub-scenario for demand, the departure time, distribution, modal split and route choice are defined. The demand model analysis is subdivided into the following sub-models:

- generation, with emergency or pre-arranged approach, in relation to the time gap available between the time when the event is perceived by the public decision maker and the time when the event occurs;

- departure time, with free (with or without user information on the system configuration) or constrained departure;

- distribution towards assembly centre, free (with or without user information on the system configuration) or constrained;

- modal split with different choice sets in relation to the alternatives: pedestrians towards a nearby assembly centre, cars towards an assembly centre further away, using a special safety 
transport mode for particular categories of disadvantaged users such as the sick, the elderly, security transport mode, prisoners, etc.;

- route choice, free (with or without user information on the system configuration) or constrained.

The supply-demand interaction model is studied in terms of an assignment model that simulates how the transportation system works, where supply and demand models are defined. In relation to the hypothesis on traffic flow, the interaction models can be:

- static or pseudo-dynamic with the hypothesis of stationary flow;

- within-day dynamic with the hypothesis of non-stationary flow.

5. The fifth phase involves the evaluation of exposure and risk acceptance levels. Exposure evaluation derives from the costs and flows on the network, carried out from the supply-demand interaction model and an exposure evaluation model. Exposure, together with the probability and vulnerability, allows us to evaluate the risk level and verify that it is acceptable or if a new scenario has to be evaluated. The risk level is evaluated with eqn (5) or eqn (7).

\subsection{Simulation problems}

The supply-demand interaction models need: the definition of a supply scenario and supply models; the definition of a demand scenario and some demand models; and supply-demand interaction analysis. The models can be considered as constraints of the design ('what to') models or as the simulation ('what if') models used to evaluate a predefined evacuation scenario.

Transport supply is represented by a graph consisting of an ordinate pair of sets, a set of elements called nodes $(i$ or $j)$ and a set of pairs of nodes $(i, j)$ called arcs or links.

The link cost vector, $\mathbf{c}$, is defined as a vector whose generic component $c_{i j}$ consists of the transport cost (generalized) on link $i j$. To each link may be associated a link flow $f_{i j}$ (f being the link flow vector) which represents the average number of users who, in the time unit, uses the link. The flow vector can only assume values belonging to its feasibility set denoted by $S_{\mathrm{f}}$. The scalar function $c_{i j}(\mathbf{f})$, which allows us to calculate the average transport cost of each link corresponding to a link flow vector, is called a cost function and may be either separable or nonseparable. In the former case, the cost of a link depends exclusively on the flow relative to the link itself:

$$
c_{i j}(\mathbf{f})=c_{i j}\left(f_{i j}\right)
$$

However, in the latter case, it depends on the flow on more than one link:

$$
\mathbf{c}=\boldsymbol{c}(\mathbf{f})
$$

Network flows are influenced by exogenous or endogenous events that affect network characteristics and the quality of service that the system normally offers [9].

In transport networks, various functions may be used to relate travel time $c$ or velocity $v$ on the link to vehicle flow $f$, capacity $C$ and other characteristics. One of the most commonly used functions is the Bureau of Public Roads (BPR) function, which relates travel time to zero flow $t_{0 i j}$ of a link $i j$, vehicle flow $f_{i j}$ on the link and capacity $C_{i j}$ to travel time $t_{i j}$ (in all the symbols, $i j$ is not reported for the sake of simplicity):

$$
t=t_{0}\left[1+\alpha(f / C)^{\beta}\right]
$$

$\alpha$ and $\beta$ are parameters greater than zero to calibrate. 
For queuing at junctions, Doherty's adaptation of Webster's formula is generally used (in all the symbols, $i j$ is not reported for the sake of simplicity):

$$
\begin{gathered}
t_{\mathrm{w}}=0.5 C l(1-\mu)^{2}+(0.55 \mu S) f /(\mu S-f) \quad \text { if } f \leq 0.95 \mu S \\
t_{\mathrm{w}}=a+b f / \mu S \quad \text { if } f>0.95 \mu S
\end{gathered}
$$

where $C l$ is the length of fictitious or real signal cycle; $\mu$ is the ratio between green time and cycle time; $S$ is the saturation flow; $a=0.5 C l(1-\mu)^{2}-198.5 / \mu S ; b=220 / \mu S$.

The traffic flow functions can be derived from the function that gives the speed $v_{i j}$ on a link $i j$ in relation to the density $k_{i j}$ on the same link. Some of the models proposed in the literature are (in all the symbols $i j$ is not reported for the sake of simplicity):

$$
\begin{array}{ll}
\text { Greenberg } & v=-v_{0}^{*} \ln \left(k / k_{\mathrm{L}}\right) \\
\text { Underwood } & v=v_{0} e^{-k / k_{0}} \\
\text { Drake } & v=v_{0} e^{-\left(k / k_{0}\right)^{2} / 2}
\end{array}
$$

where $\mathrm{v}_{0}$ is the free flow speed; $v_{0}^{*}$ is the free flow speed with density near zero; $k_{\mathrm{L}}$ is the jam density; $k_{0}$ is the density with flow equal to capacity.

The use of demand models in simulating an evacuation plan involves various problems connected with the many cases that may lead to a state of emergency. Below are considered two decisional units: on the one hand the citizen user who has to decide in some cases how to act, and on the other the public decision maker (prefect, mayor, disaster manager, security and safety operational forces, etc.) who may impose or advise some user choices. The public decision makers must predict the effects that implementation of their measures have on the system and how these measures may interact with the individual reactions of the users served by the transport system in question.

Average demand, with its main characteristics in a certain reference period, may be obtained with a system of demand models that allows us to associate to a given system of activities and transport supply, the average number of trips with certain characteristics and in a reference period. In all choice models, we need to define the set of alternatives considered by the public decision maker and the user, as well as the type of choice models considered [6,9].

The choice model from a given choice set, in the context of random utility models, assumes that a generic user, travelling between an origin-destination pair $(r, s)$, associates to each alternative $k$ belonging to the set $K_{\text {od }}$ of available alternatives connecting the origin/destination (O/D) pair, a perceived utility $U_{k, r s}$ which may be expressed as:

$$
U_{k, r s}=V_{k, r s}+\varepsilon_{k, r s} \quad \forall k \in K_{\mathrm{od}}
$$

The term $V_{k, r s}$ denotes the average, or systematic, utility of alternative $k$, while the random residual $\varepsilon_{k, r s}$ is usually assumed to include perception errors of the decision maker as well as the modelling approximation of the analyst.

Different specifications can be used for term $V_{k, r s}$. A possible specification is:

$$
V_{k, r s}=\Sigma_{1} \alpha_{1} X_{1}-\Sigma_{\mathrm{m}} \beta_{\mathrm{m}} Y_{\mathrm{m}}
$$

where $\alpha_{1}$ and $\beta_{\mathrm{m}}$ are parameters greater than zero to calibrate; $X_{1}$ are user utility attributes; $Y_{\mathrm{m}}$ are user disutility attributes.

The subscript $r$ should be used in particular to indicate the generic trip origin and $s$ the assembly centre, $d_{r s}$ being the number of users travelling between the origin and the assembly centre pair $(r, s)$. 
Table 1: Example of attribute specification for choice models.

\begin{tabular}{lll}
\hline Choice dimension & Utility attributes $X_{l}$ & Disutility attributes $Y_{m}$ \\
\hline Generations & $\begin{array}{l}\text { Resident, vehicle owner, number } \\
\text { or people in family, sick }\end{array}$ & \\
Accessibility, resident & $\begin{array}{l}\text { Congestion (or inclusive utility in } \\
\text { inferior levels) }\end{array}$ \\
Distribution & Resident, worker, students & $\begin{array}{l}\text { Distance, travel time (or inclusive } \\
\text { utility in inferior levels) }\end{array}$ \\
Modal split & Vehicle owner, available mode & $\begin{array}{l}\text { Travel time (or inclusive utility in } \\
\text { inferior levels) }\end{array}$ \\
Route choice & & Travel time \\
\hline
\end{tabular}

Some O/D pairs are relative to the demand of users who have to continue working even in emergency conditions. The demand vector $\mathbf{d}$ (whose generic element is $d_{r s}$ ) may assume values belonging to its feasibility set denoted by $S_{d}$.

The demand can be evaluated with the use of different models. In this paper, we use an update of standard sequence of sub-models: a generations model supplying trips originating from a certain zone in the study area; a departure time model supplying the number of users leaving in each time slice; a distribution model supplying the number of users reaching a particular assembly centre from an origin zone; a modal split model supplying the number of users of a given transport mode from a certain origin to a certain assembly centre; a route choice model supplying the percentage of users who use a certain route between a fixed origin and an assembly centre with a transport mode. In Table 1 an example of attribute specification of the different models is reported.

A model of supply-demand interaction allows us to simulate the way in which the demand uses the supply system, producing flows on network links that in turn generate travel costs. The system configuration, simulated through models, depends on user behavioural hypotheses (attributes used, distribution of random residuals, level of information available on supply, quality of information and constraints to follow the information).

Demand-supply interaction in the system, in the case of evacuation, cannot be tackled by using static assignment models directly with stationary flow. On the other hand, the use of dynamic assignment models, in a non-stationary approach, requires many parameters to calibrate and there is often a lack of complete data regarding transport systems on which to calibrate the models. An intermediate approach, named pseudo-dynamic approach, has to be considered where the time is divided into intervals and the flow is considered stationary in each interval. In this approach, each interval can be simulated with a static approach where the initial system characteristics are obtained from the simulation in the previous interval.

The main static (with stationary flow) and within-day dynamic (with non-stationary flow) models that can be used for analyzing demand-supply interaction are reported below.

In static models, three approaches can be used:

- the stochastic equilibrium approach, i.e. the use of static assignment models with stochastic user behaviour, by which there is more or less correct reproduction of user information, non-perfect respect of impositions and alternative choice behaviour;

- the deterministic equilibrium approach, i.e. the use of static assignment models with deterministic user behaviour, by which complete user knowledge of the system is reproduced; 
- the system optimum (SO) approach, i.e. the use of static assignment models with user behaviour imposed by the public decision maker, .

In within-day dynamic models [12-14], it is hypothesized that the flow variables may have a variability not only in space but also in time. There may be three aggregation levels in representing the flow: aggregate flow parameters; analysis of homogeneous vehicle groups (vehicle packages); and analysis of individual vehicles. Macroscopic models use a continuous representation of the flow, as if it were a fluid, in a way similar to that of hydrodynamic theory. Microscopic and mesoscopic models employ a desegregate representation of individual (microscopic) or group (mesoscopic) user behaviour. Model outputs either refer to the individual user or a group of users. In the case of macroscopic models, the individual vehicle (or a group of vehicles) is not monitored directly, while in the other models vehicle trajectories are directly monitored. In evacuation problems, macroscopic models are generally not used.

Some systems based on this approach are DynaMIT [15] developed in the MIT of Boston, DYNASMART [16] developed in the University of Texas at Austin and University of Maryland, VISSIM [17] developed by PTV.

\subsection{Design problem}

The 'what to' (or design) approach consists in defining some objectives and finding the system configurations as an optimum of the objective function. In fact, just for a subset of transportation design problems, it is possible:

- to formulate a model for which solution existence and uniqueness are guaranteed;

- to develop an exact solution algorithm that converges in the unique solution in an acceptable computation time.

The design methodology is developed to seek an optimal configuration of the transportation system with respect to some objectives and considering user behaviour [18]. It consists in determining the optimal configuration of the urban network elements (topology, capacity, etc.) and demand characteristics (time departure, distribution, etc.), with respect to a set of criteria.

This paper makes reference to the formulation proposed in [19]. The problem is defined as a minimization of an objective function $\Phi(\mathbf{w}, \mathbf{f})$ subject to supply, demand, supply-demand interactions and budget constraints:

$$
(\mathbf{w}, \mathbf{f})^{*}=\underset{\mathbf{w}, \mathbf{f}}{\arg \min } \Phi(\mathbf{w}, \mathbf{f})
$$

where $\mathbf{f}$ is the vector of link flow; $\mathbf{w}$ is the vector of control variables. Common elements can be found in the method proposed for transportation system design for objective categories for each group of stakeholders (decision makers), control variables, constraints and solution generations.

Three categories of objectives can be considered:

- (1) system analysts seek to implement a decision support system in order to evaluate in a short time the effect of the strategy that has to be implemented in the system;

- (2) public decision maker and (3) operational forces seek to reduce system management costs, maximize the system utility (safety, security) and seek to reduce traffic incidents.

The control variables concern the supply and demand configuration.

Three main constraints are considered, representing user behaviour in path choice, system capacity and demand configuration. Users seek to reduce their travel time, stress, congestion and pedestrian paths, and seek to maximize their utility and safety (try to reach the safe point or assembly centres 
in a minimum time). User behaviour is simulated with traffic assignment. Traffic assignment can be carried out with deterministic or stochastic user behaviour.

In relation to the 'what to' approach, only topological and capacity design models have been proposed in the literature [18-22]. The network design models and the solution algorithms deal distinctly with the problem of network design for road transport and for transit services. In the former case, an optimal configuration of links and intersection capacities is generated, while in the latter an optimal configuration in terms of topology and optimal frequency of transit lines is carried out. The 'what to' approach can be specified in relation to the system that has to be designed (metropolitan area, city, building, transportation means, industrial area). Some variables have to be specified in each case.

The prime objective to consider for designing a transportation system in emergency conditions concerns the user, since all actions have to be implemented to save lives. The aim is to minimize the societal risk $R_{L_{E}, T, \Delta}$.

The decision variables of the problem are those that are relative to the transportation system configuration (demand and supply) and the public decision maker. The control variables can involve supply (network design problem) and/or demand (demand management problem).

For supply, the design variables that can be considered are:

- $\mathbf{w}_{\mathrm{tc}(\mathbf{u})}$ links the topology (t) and the capacity (c) vector for users (u); it defines the direction of each link and the junction strategy for users dividing the vector into two parts: one relative to the topology and the other relative to the capacity;

- $\mathbf{w}_{\text {tc(b) }}$ links the topology and the capacity vector for breakdown (b) services; it defines the direction of each link and the junction strategy for breakdown services;

- $\mathbf{w}_{\mathbf{b}}$ the breakdown services vehicle vector defines the category and the number of vehicles that have to be used for each service;

For demand, the design variables that can be considered are:

- $\Delta \mathbf{h}$ vector of departure time duration; each element reports the duration of the corresponding time slice;

- $\mathbf{d}_{\text {TIME(h),D }}$ demand vector relative to the distribution level with the constraint on the departure time; each element is relative to a specific O/D pair $(r, s)$ and reports the total trips between $r$ and $s$ for each departure time slice $h$;

- $\mathbf{D}_{\text {Time, }}=\left\{\mathbf{d}_{\text {Time(1),D }} ; \ldots ; \mathbf{d}_{\text {Time(h),D }} ; \ldots ; \mathbf{d}_{\text {Time(nh),D }}\right\}$ distribution matrix in the different departure time slice; if only one time slice is considered, the matrix $\mathbf{D}_{\text {TIME,D }}$ coincides with the vector $\mathbf{d}_{\mathbf{D}}$; the demand management problem of $\mathbf{d}_{\mathbf{D}}$ is a particular case of the $\mathbf{D}_{\mathbf{T I M E}, \mathbf{D}}$ demand management problem;

- $\mathbf{d}_{\mathbf{D}}$ demand vector relative to the distribution level without the constraint on the departure time; each element is relative to a specific O/D pair $(r, s)$ and reports the total of trips between $r$ and $s$.

In formal terms, the evacuation design problem, specifying eqn (8), can be formulated as:

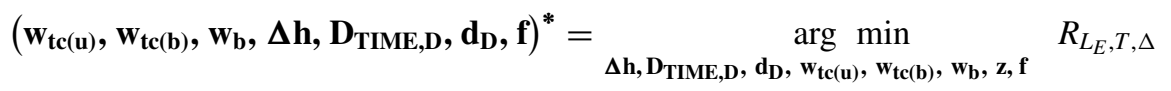

The constraints are relative to the decision variables that have to belong to their choice set definition. Another set of constraints regards traffic flow that has to be obtained with supply-demand interaction models that consider user behaviour. This is reported in Section 3.2. 


\section{PATH DESIGN IN EMERGENCY CONDITIONS}

In this section, we consider that the risk has to be evaluated and reduced when an event happens or is bound to happen in the short term and an evacuation plan has to be generated (also in real time). Hence the risk is formulated taking the following hypotheses into account:

- an event happens with a predefined intensity level and in a homogeneous area with respect to the event;

- vulnerability $V(x, y)$ is constant.

An analysis of the effect of frequency or probability and vulnerability on the risk function may be found in papers belonging to other research areas. For frequency or probability, examples of events and theories are: for earthquakes, geotechnical and geologic theory; for chemical effects, chemistry theory; and for terrorist attacks, policing and security theories. For vulnerability, some examples of structures and theories are: for building vulnerability, construction theory; for vehicles, mechanical theory.

Exposure in the literature has not been studied with a quantitative approach. Nowadays, exposure reduction with evacuation design has to be developed in transportation science.

Starting from the hypothesis given, a general formulation for designing transportation systems can be developed. The aim is to solve eqn (9), minimizing the risk function $R$ as defined in eqn (5).

It is supposed that for each event $E$, with intensity level in the range $L_{E}$, in an area $T$ :

- the vulnerability function $V$ cannot be modified and is given equal to $V$ (constant values in $L_{E}$ and $T$ );

- the exposure function $N$ is constant with respect to $x$ in the range $L_{E}$ and equal to the function $N^{*}(y)$

- the probability function $p$ cannot be modified and is given as equal to the constant value $p_{0}$ in $L_{E}, T$ and $\Delta$.

Considering these hypotheses, societal risk $R$ reported in eqn (5) is:

$$
\begin{aligned}
R_{L_{E}, T, \Delta} & =V p_{0}\left(\int_{x \in L_{E}} \int_{z \in \Delta} \mathrm{d} x \mathrm{~d} z\right) \int_{y \in T} N^{*}(y) \mathrm{d} y /\left(\int_{x \in L_{E}} \int_{y \in T} \int_{z \in \Delta} \mathrm{d} x \mathrm{~d} y \mathrm{~d} z\right) \\
& =V p_{0}\left(\int_{y \in T} N^{*}(y) \mathrm{d} y\right) /\left(\int_{y \in T} \mathrm{~d} y\right)
\end{aligned}
$$

with the hypothesis that the function $N^{*}(y)$ allows the triple integral to be separated into three single integrals.

For $N^{*}(y)$, in relation to the optimization problem considered, different specific exposure functions can be defined as objective functions, to identify evacuation measures in transportation systems.

The area system and the transportation supply relative to the area $\mathrm{T}$ can be discretized in a finite number of homogeneous areas with respect to exposure, represented by:

- in the links representing sections of road, relative area activities and buildings giving onto them (if a road is not homogeneous in terms of exposure, the sections are represented with different links in series);

- nodes representing

- the junctions between links;

- the fictitious points $(r)$ where the origin of the user trip towards the assembly centres is assumed to be concentrated;

- the assembly centres $(s)$ (safe points). 
As described in Section 3.1, the transportation supply is represented by a graph, composed of links and nodes, and a vector $\mathbf{c}$ of link costs and a vector $\mathbf{f}$ of link flows associated to the links.

We may associate to each loop-less path $\mathrm{k}$ of the graph connecting the origins (fictitious points) to the assembly centres:

- a path flow $h_{k}$ (h being the flow path vector) which represents the average number of users who, in the time unit, use path $k$ from their origin (in a fictitious point) to their destination (in an assembly centre);

- a path cost $g_{k}$ (g being the cost path vector) which represents the average cost of users who, in the time unit, use path $k$ from their origin (in a fictitious point) to their destination (in an assembly centre).

The path cost $g_{k}$ is the sum of the link cost $c_{i j}(\mathbf{f})$ that belong $(\in)$ to path $k$ :

$$
g_{k}=\sum_{i j \in k} c_{i j}(\mathbf{f})
$$

Considering the binary index $\delta_{i j, k}$, that is 1 if link $i j$ belongs to path $k$ and 0 otherwise; the path cost $g_{k}$ can also be written as the sum of the cost $\mathrm{c}_{i j}(\mathbf{f})$ of links $i j$ that belong to path $k$ :

$$
g_{k}=\sum_{i j} \delta_{i j, k} c_{i j}(\mathbf{f})
$$

The link flow $f_{i j}$ is the sum of the flow $h_{k}$ relative to the path $k$ that contains $(\supset)$ the link $i j$ :

$$
f_{i j}=\sum_{k \supset i j} h_{k}
$$

Considering the binary index $\delta_{i j, k}$ the link flow $f_{i j}$ can also be written as the sum of the flow $h_{k}$ of paths $h$ that contain the link $i j$ :

$$
f_{i j}=\sum_{k} \delta_{i j, k} h_{k}
$$

In this context, the integral reported in eqn (10) to assess the risk can be transformed in a sum relative to the homogeneous elements (nodes and links). The exposure $N^{*}(y)$ in $T$ can be evaluated separately for each homogeneous zone $r$ as the sum of the cost for all users to reach the safe points $s$, departing from $\mathrm{r}$ and considering all the paths $(\Rightarrow)$ that connect $r$ and $s$ :

$$
N^{*}(r)=\sum_{s} \sum_{k \Rightarrow r s} g_{k} h_{k}
$$

Global exposure evaluated in $T$ reported in eqn (10) $\left(\int_{y \in T} N^{*}(y)\right.$ dy) can be expressed as the sum of $N^{*}(r)$ in all the homogeneous zones $\mathrm{r}$ :

$$
\int_{y \in T} N^{*}(y) \mathrm{d} y=\sum_{r} N^{*}(r)=\sum_{r} \sum_{s} \sum_{k \Rightarrow r s} g_{k} h_{k}
$$

Considering the paths $k\left(\sum_{k \Rightarrow r s}\right)$ that connect all the origins $r\left(\sum_{r}\right)$ to all the destinations $s\left(\sum_{s}\right)$ is equivalent to considering all the paths $k\left(\sum_{k}\right)$. The previous equation can be written as:

$$
\int_{y \in T} N^{*}(y) \mathrm{d} y=\sum_{k} g_{k} h_{k}
$$


The global exposure $\sum_{k} g_{k} h_{k}$, considering eqn (11), can be written as $\sum_{k} \sum_{i j} \delta_{i j, k} c_{i j}(\mathbf{f}) h_{k}$ and also in the form $\sum_{i j}\left(\sum_{k} \delta_{i j, k} h_{k}\right) c_{i j}(\mathbf{f})$. Considering eqn (12), the global exposure can be written as $\sum_{i j} c_{i j}(\mathbf{f}) f_{i j}$. The global exposure can also be written as:

$$
\int_{y \in T} N^{*}(y) \mathrm{d} y=\sum_{i j} c_{i j}(\mathbf{f}) f_{i j}=\mathbf{c}^{\mathrm{T}} \mathbf{f}
$$

The societal risk reported in eqn (10) finally is:

$$
R_{L_{E}, T, \Delta}=V P\left(\mathbf{c}^{\mathrm{T}} \mathbf{f}\right) /\left(\int_{y \in T} \mathrm{~d} y\right)
$$

Other risk exposures can be considered, such as the evacuation time defined as the time at which the last vehicle exits from the network.

In a transportation system and with the previous hypotheses (constant values of the functions $V$ and $p$, and constant value of function $N$ in $L_{E}$ ), minimization of the risk function $R$ is expressed as minimization of the exposure function $N^{*}(y)$ with the integral throughout area T. The optimization problem (eqn 10) consists in obtaining the flows $\mathbf{f}^{*}(\arg \min )$ that minimize the risk function (eqn 15):

$$
\mathbf{f}^{*}=\arg \min _{\mathbf{f} \in S_{\mathrm{f}}} R_{L_{E}, T, \Delta}=\arg \min _{\mathbf{f} \in S_{\mathrm{f}}} V p_{0}\left(\mathbf{c}^{\mathrm{T}} \mathbf{f}\right)\left(\int_{y \in T} \mathrm{~d} y\right)
$$

From a transport point of view, the terms $V$ and $P$ are assumed as constants, as other research areas study the problem to reduce them. The term $\int_{y \in T} \mathrm{~d} y$ is the size of $T$ and is constant. The optimization problem eqn (15) is equivalent to the following:

$$
\mathbf{f}^{*}=\arg \min _{\mathbf{f} \in S_{\mathrm{f}}} R_{L_{E}, T, \Delta}=\arg \min _{\mathbf{f} \in S_{\mathrm{f}}} \mathbf{c}^{\mathrm{T}} \mathbf{f}
$$

Risk $R_{L_{E}, T, \Delta}$ can be valued considering the optimal flow $\mathbf{f}^{*}$ in eqn (14).

The problem reported in eqn (16) is a formulation in terms of design (or 'what to') approach where an objective function (in this context $\mathbf{c}^{\mathrm{T}} \mathbf{f}$ represents the sum of the cost for all users to reach the safe points) is minimized. At the minimum for the objective function, the path and link flows and costs are obtained. The users carry out the flows and the costs with an optimum set of path choice. Users in order to minimize global exposure must follow the optimal path.

The problem reported in eqn (16) is called in the literature SO and was first formulated in [23].

The conditions for the existence and uniqueness of the solution for eqn (16) are relative to the cost function that has to be continuous, differentiable and in monotone. These conditions are demonstrated in [9].

In some cases vulnerability may not be constant. The problem can be solved with different levels of vulnerability; different optimal solutions can be obtained. This is possible for example if the risk and the optimal system configuration have to be defined in real time after an event happens on the system. In this context, the infrastructural configuration is defined in real time and the new optimal configuration can be generated.

\section{EXPERIMENTATION}

Different models and procedures can be applied to solve the problem (eqn 9). Exact methods can be applied for solving the system optimum to find the optimal paths and in very small systems to design the link layout. In real systems, heuristic approaches or the simulation approach in different scenarios chosen by the analyst have to be applied. 


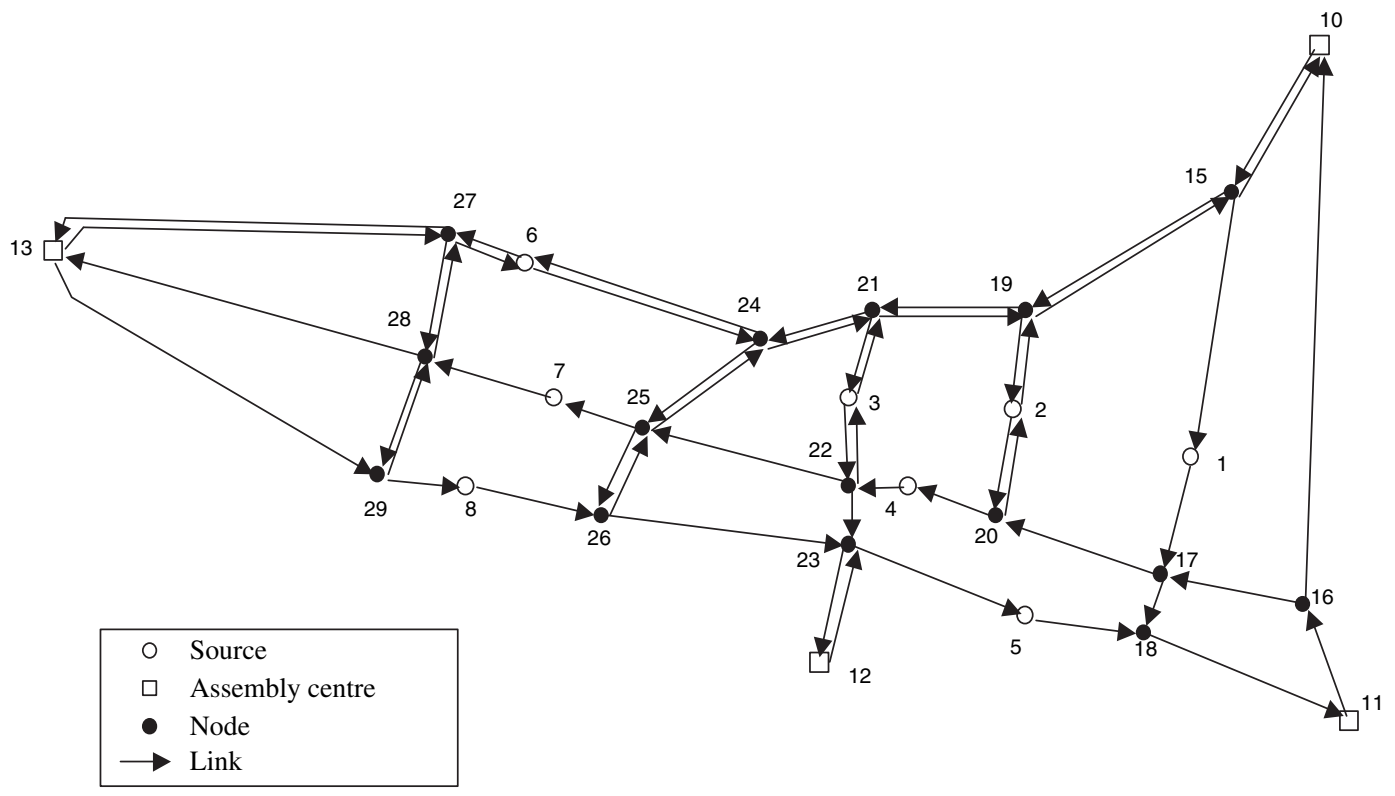

Figure 3: Graph of the town, Zafferana Etnea.

In this section, these methods are applied to the town of Zafferana Etnea, Italy. The town has about 9,000 inhabitants and was chosen because it has a similar number of potential evacuees to the majority of cases in real evacuations, where entire small towns, parts of a city or big buildings have to be evacuated.

The road network simulated for the town of Zafferana Etnea has (Fig. 3):

- 30 nodes ( 8 origins and 4 assembly centres named 10, 11, 12 and 13, located on the border of the area);

- 61 road links.

In all, 2,000 vehicles are considered on the system.

The 'what if' approach reported in Section 3.1 was applied in order to evaluate the best distribution considering the 'what to' objective function reported in eqn (16).

Different scenarios chosen by the analyst are tested in relation to the

- demand (distribution between assembly centres) sub-scenarios;

- supply (open assembly centres) sub-scenarios;

and for each scenario the 'what to' path solution (as described in Section 4) is obtained.

The 'what to' approach reported in Sections 3.2 and 4 is applied, considering user behaviour as a constraint and simulating it with stationary and pseudo-dynamic evolution in time. The pseudodynamic approach (described in Section 3.2) is based on static approach concepts and can be applied with commercial software, but it does not explicitly simulate interaction among vehicles and queue formation and dissipation phenomena. In evacuation problems, it is worth simulating the system by subdividing simulation time into several intervals in which flow is maintained in stationary conditions and demand, to be assigned, is compatible with supply. 
The demand could be distributed between the assembly centres in two different ways:

- uniform demand distribution: drivers are equally distributed from each origin $r$ among all the available assembly centres $s$

$$
d_{D r s}=d_{E r} / A_{\mathrm{C}}
$$

where $d_{E r}$ is the total users distributed from origin $r$ and $A_{\mathrm{C}}$ is the number of assembly centres;

- non-uniform demand distribution: drivers are distributed from origin $r$ among the available assembly centres $s$ according to their capacities $K C_{s}$ and their distance $D C_{r s}$ from the origin considering a gravitational model

$$
d_{D r s}=d_{E r}\left[K C_{s}^{\beta 1} D C_{r s}^{-\beta 1}\right] /\left[\sum_{s} \mathrm{KC}_{s}^{\beta 1} \mathrm{DC}_{r s}^{-\beta 2}\right]
$$

where $\beta_{1}$ and $\beta_{2}$ are two parameters greater than zero.

The supply is analyzed considering four scenarios for system vulnerability in relation to the following characteristics:

- assembly centres 11, 12 and 13 closed, with uniform demand distribution;

- assembly centres 11, 12 and 13 closed, with non-uniform demand distribution;

- assembly centres 12 and 13 closed, with non-uniform demand distribution;

- assembly centre 12 closed, with non-uniform demand distribution.

The four scenarios are considered with the same number of users to be evacuated and with different hypotheses in network vulnerability. In the first two scenarios, it is supposed that the vulnerability is high and three on four assembly centres are impossible to reach. In the third scenario, it is supposed that only two assembly centres cannot be reached; and in the last scenario, it is supposed that only one assembly centre cannot be reached.

In order to analyze and compare network performances, the following objectives are defined:

- $\quad N_{1}(T)$, total travel time on the network (eqn 17);

- $N_{2}(T)$, evacuation time or time at which the last vehicle exits from the network.

Table 2 presents the values of the objectives for one scenario, considering the two demand distributions among the assembly centres and taking into account the time for queue formation and dissipation. Comparing the uniform and non-uniform demand distribution, the values of the indicators are evidently greater in the uniform demand distribution. As previously stated, this confirms the need to modify not only supply but also demand, such as the demand distribution dimension.

The quantitative representation of the curve between vulnerability and exposure for the town of Zafferana Etnea is reported in Fig. 4. In the figure, we consider the time to evacuate all users as indicators for exposure, and the number of non-connected assembly centres as indicators of vulnerability. The four scenarios were tested and reported in Fig. 4. From the first to the second scenario, only evacuation measures are designed. From the second to the third and from the third to the fourth scenarios, it is supposed that a new infrastructural configuration is present (resistance measure) and evacuation measures are designed. It is evident that the increase in vulnerability also generates an increase in exposure and, in general, an increase in risk, given that the probability (or frequency) that an event occurs is independent of vulnerability and exposure.

The four scenarios give interesting information at two levels: in the specific case of Zafferana Etnea and in the more general case. 
Table 2: Indicators for the defined scenarios and relative variations: pseudo-dynamic approach.

\begin{tabular}{lccccc}
\hline & \multicolumn{4}{c}{ Demand distribution } \\
\cline { 2 - 5 } & \multicolumn{2}{c}{ Uniform } & & \multicolumn{2}{c}{ Non-uniform } \\
\cline { 2 - 5 } \cline { 5 - 6 } Closed assembly centres & $N_{1}(T)(\mathrm{min})$ & $\begin{array}{c}N_{2}(T) \\
\text { (Vehicles hours) }\end{array}$ & & $N_{1}(T)(\mathrm{min})$ & $\begin{array}{c}N_{2}(T) \\
\text { (Vehicles hours) }\end{array}$ \\
\hline $11,12,13$ & 46 & 235 & & 27 & 169 \\
\hline
\end{tabular}

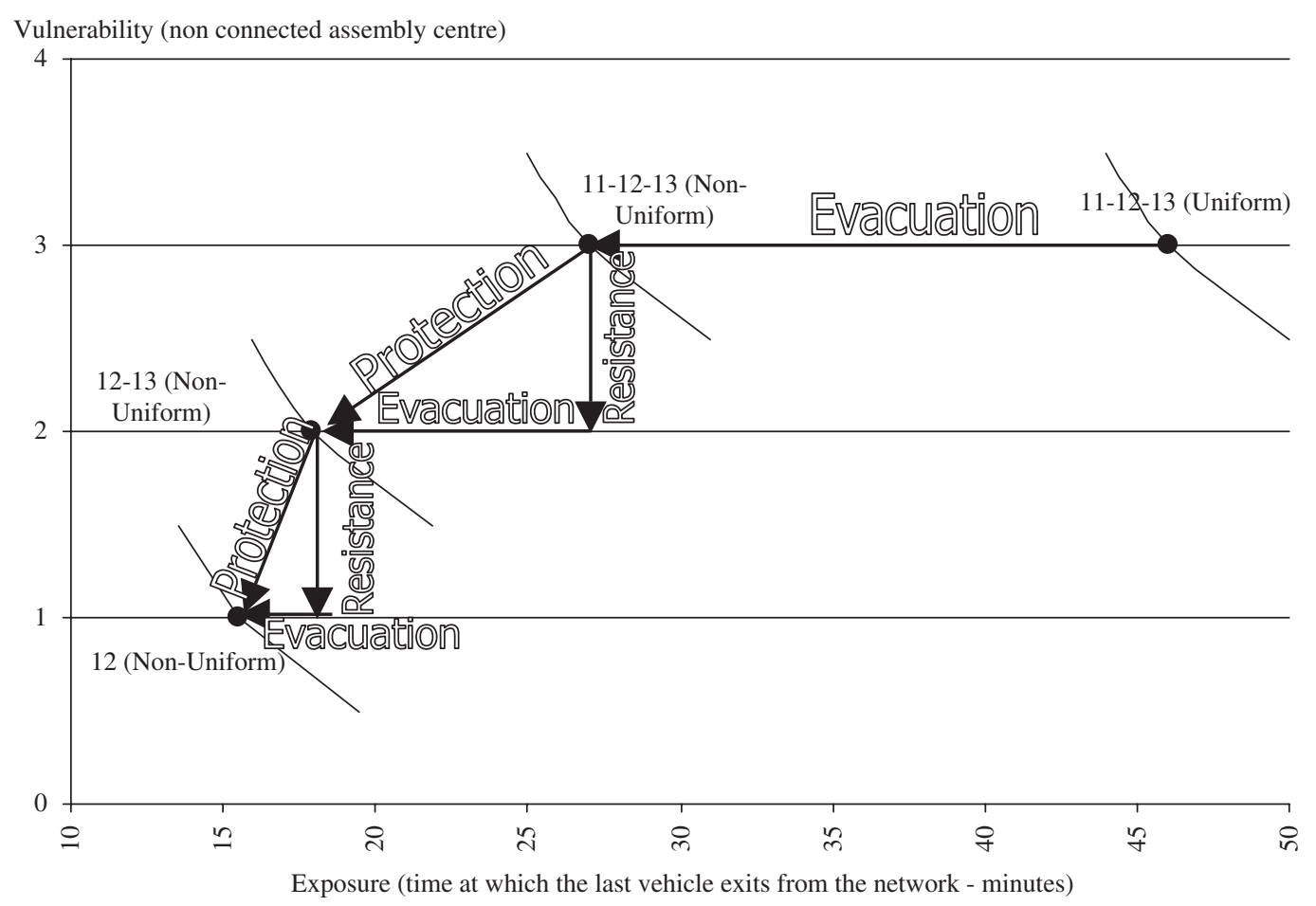

Vulnerability $=$ Number of non-connected assembly centres $v$ s. Exposure = Time at which the last vehicle exits from the network

Figure 4: Numerical results for vulnerability and exposure in the town of Zafferana Etnea.

In Zafferana Etnea it may be observed that in the best case considered, where only assembly centre 12 is not reachable and in due consideration of evacuation design, in about 15 minutes the village is evacuated. Where assembly center 13 is also not reachable and given the evacuation design, the evacuation time is about 18 minutes. Supposing that also assembly centre 13 is not reachable (worst case), with evacuation design, the evacuation time is 27 minutes; and without evacuation design, the evacuation time is 46 minutes.

These considerations can be extended to more general problems of evacuation design. Clearly, evacuation design reduces evacuation time. This reduction is high in systems with higher vulnerability and is lower in systems with lower vulnerability. In the case of Zafferana Etnea in the worst case 
(high vulnerability, three non-connected assembly centres) the reduction in the evacuation time is about $40 \%$, in the best case (low vulnerability, one non-connected assembly centre) the reduction in the evacuation time is about $15 \%$.

\section{CONCLUSIONS AND INDICATIONS FOR RESEARCH DEVELOPMENTS}

In this paper, a general model for risk evaluation in transportation systems is proposed and vulnerability and exposure are explained. Methods for evacuation are proposed for simulating and designing evacuation in a road urban network system in emergency conditions. The design model is solved with 'what if' and 'what to' procedures testing different scenarios. The models are applied in a small real system.

Research for the analysis and modelling of transportation systems in emergency conditions requires further studies in supply, demand, supply-demand interaction and design. In emergency conditions, there is the need to develop new methods and rearrange standard procedures such as: network vulnerability analysis in terms of the 'safety coefficient' of the supply system in relation to events with different levels of danger and different probabilities of fulfilment; specification and calibration of link cost functions to use in system simulation in over-saturation conditions in the periods analyzed; and specification and calibration of demand models for the different choice levels for users and for the public decision maker. In this paper, it is evident that the definition of the best scenario emerges only from the simulation of predefined scenarios, taking into account supply, demand and their interaction. The management of emergency conditions and evacuation of an urban area must necessarily be supported by quantitative analyses.

\section{REFERENCES}

[1] Goldblatt, R., Development of evacuation time estimates for the Davis nuclear power station. Technical Report KLD, 329, 1993.

[2] Russo, F. \& Vitetta, A., The road network design problem to improve the safety during exogenous flow perturbations. Proceedings of the 29th ISATA Conference, Florence, 1996.

[3] Goldblat, R., Evacuation planning. Human factor and traffic engineering perspectives. Proceedings of the European Transport Conference, Strasbourg, 2004.

[4] University of Maryland, Fire and Rescue Institute http://www.mfri.org, 2005.

[5] Di Gangi, M., Luongo, A. \& Polidoro, R., Una procedura di carico dinamico per la valutazione dei piani di evacuazione. Atti del $2^{\circ}$ seminario scientifico su Metodi e Tecnologie dell'Ingegneria dei Trasporti, Università di Reggio Calabria, Franco Angeli, Milan, 2000.

[6] Ben Akiva, M. \& Lerman, S., Discrete Choice Analysis: Theory and Application to Travel Demand, MIT Press: Cambridge, MA, 1985.

[7] Sheffi, Y., Urban Transportation Networks, Prentice Hall: Englewood Cliff, NJ, 1985.

[8] Train, K., Discrete Choice Methods with Simulation, MIT Press: Cambridge, MA, 2003.

[9] Cascetta, E., Transportation Systems Engineering: Theory and Methods, Kluwer Academic Publishers: Dordrecht, 2001.

[10] CCPS, Guidelines for Chemical Transportation Risk Analysis, American Institute of Chemical Engineers: New York, 1995.

[11] Health and Safety Commission, Major hazard aspects of the transport of dangerous substances. Report and appendices, HMSO, London, 1991.

[12] Ben Akiva, M. \& De Palma, A., Dynamic models of transportation networks. Proceedings of the 15th PTRC Summer Annual Meeting, University of Bath, England, 1987.

[13] Cascetta, E. \& Cantarella, G.E., Modelling dynamics in transportation networks. Journal of Simulation Practice and Theory, 1, pp. 65-91, 1993. 
[14] Cascetta, E., \& Cantarella, G.E., A Day-to-Day and Within-Day dynamic stochastic assignment model. Transportation Research A, 25, pp. 277-291, 1990.

[15] Ben-Akiva, M. Bierlaire, M., Koutsopoulos, H. \& Mishalani, R., DynaMIT: a simulation based system for traffic prediction and guidance generation. Proceedings of the TRISTAN III, San Juan, 1998.

[16] Mahmassani, H.S. \& Hawas Y., Data requirement for development, calibration of dynamic traffic models, and algorithms for ATMS/ATIS. Proceedings of the 76th Annual Meeting of the Transportation Research Board, Washington, DC, 1997.

[17] VISSIM User Manual, PTV AG, Karlsruhe, Germany, 2001.

[18] Transportation Research B, 35, Pergamon, 2001.

[19] Magnanti, T.L. \& Wong, R.T., Network design and transportation planning: models and algorithms. Transportation Science, pp. 1-55, 1984.

[20] Billheimer, J.W. \& Gray, P., Network design with fixed and variable cost elements. Transportation Science, 7, pp. 49-74, 1973.

[21] Ceder, A. \& Wilson, N.H.M., Bus network design. Transportation Research B, 20, 1986.

[22] Poorzahedy, H. \& Turnquist, M.A., Approximate algorithms for the discrete network design problem. Transportation Research B, 16, pp. 45-55, 1982.

[23] Wardrop, J. G., Some theoretical aspects of road traffic research. Proceedings of the Institution of Civil Engineers, Part II, Vol. 1, pp. 325-378, 1952. 